\title{
Hardware-software implementation of the compression and transmission of seismic data
}

\author{
Leisan Sh. Murtazina, Evgeniy V. Danilov, Evgeniy Yu. Ryabchenko, Oleg N. Sherstyukov \\ Department of Radiophysics, Institute of Physics, Kazan Federal University, Kazan, Russia
}

\begin{abstract}
A real-time wireless network architecture for seismic data acquisition system based on multi-level radio network is proposed. The single-chip programmable transceiver Si446x that allows creating the first level of the network is considered. Physical layer with GFSK modulation in the sub-gigahertz band is considered. Data link layer implementation with generic frame structure for a network, operating in synchronous mode, is presented. An algorithm for seismic data accumulating and compressing is presented. The comparison of existing and proposed solutions is given.
\end{abstract}

\section{Introduction}

Seismic data acquisition complexes used in geological prospecting work provide concurrent transmission of signals from numerous measuring channels with typical sampling rate of $500-1000 \mathrm{~Hz}$ of 24-bit samples. Ordinarily, such data streams (12000-24000 bps per channel) are transmitted using cable technologies.

Wireless data transmission technologies are actively developed at the moment, which should reduce the total area of deforestation during cable laying. For these purposes, realtime systems are needed to quickly obtain and assess the quality of seismic data, as well as to monitor the operation of all equipment. One of the most challenging problems in the development of such systems is the suppression of considerable attenuation (the most unfavorable case is a continuous deciduous forest) in the UHF range to provide radio communication over long distances in conditions of considerable attenuation acceptable for broadband organization of radio communications [1].

The above-stated problem can be solved by using a retransferring ("message forwarding") method of data transmission, where each seismic acquisition point sends not only its own data, but also retransmits the data of the adjoining point "in a daisy chain" in the direction of the central collection point.

Since 2012, such a solution has been presented by Wireless Seismic (USA) in its RT2 complexes based on a broadband radio channel in the $2.4 \mathrm{GHz}$ ISM band [2,3]. Another solution is a SCOUT telemetry system by "SKBSP JSC" [2]. The technical characteristics of the above-mentioned complexes and the comparison with the developed architecture will be given in the conclusion. 


\section{Proposed architecture}

A multi-level telemetry system (Fig.1) using radio channels of various frequencies and modulation types [4], considered in this paper, is one of the options for implementing a real-time wireless seismic complex.

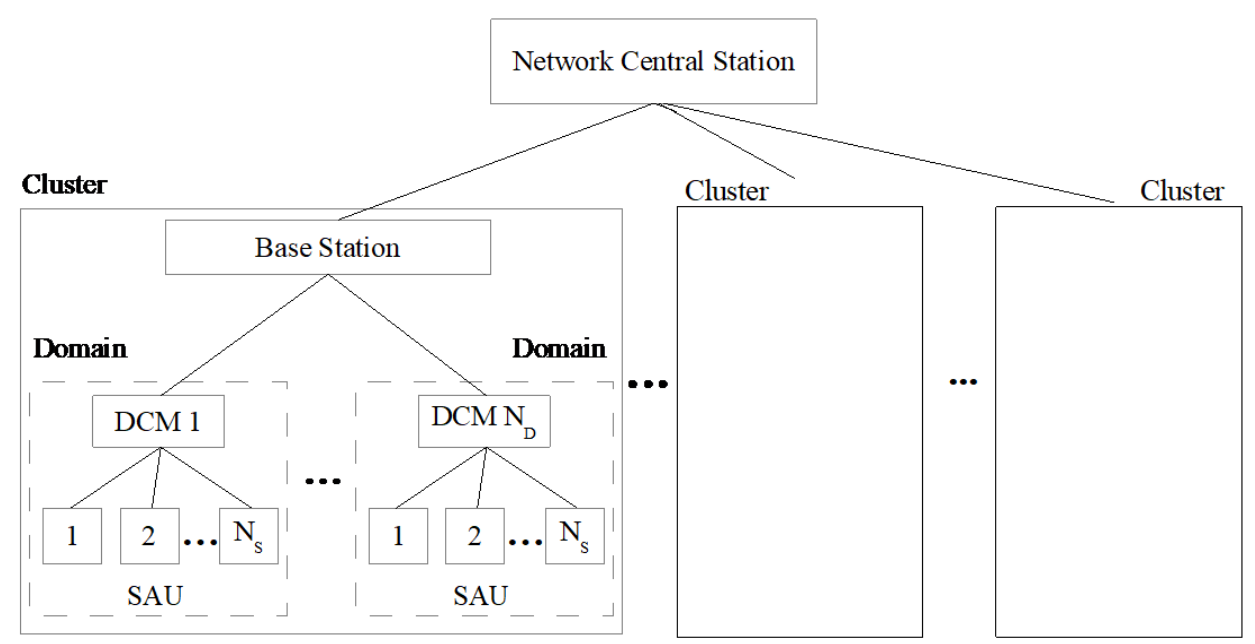

Fig. 1. Multi-level radio network architecture for seismic data acquisition system.

At the first level, data are transmitted from the field units communicated with a seismic receiver - seismic acquisition units (SAU) - to transitional repeaters, - domain coordinator modules (DCMs) via the radio channel (RCh1). The SAUs and one DCM are grouped into one domain.

The second level of the radio network is formed for transfering data from all SAUs via the radio channel 2 (RCh2) to the base station (BS). One BS forms a cluster and serves up to $\mathrm{N}_{\mathrm{D}}$ DCMs.

At the third level, data from all clusters are collected via the radio channel 3 (RCh3) or cable technologies to the network central station (NCS), which also manages the operation of the entire complex.

Equation 1 governs the total number of measuring channels, where $\mathrm{N}_{\mathrm{C}}$ is the number of clusters, upon the condition that the values $\mathrm{N}_{\mathrm{D}}$ and $\mathrm{N}_{\mathrm{S}}$ are constant for the whole complex.

$$
\mathrm{N}=\mathrm{N}_{\mathrm{C}} \cdot \mathrm{N}_{\mathrm{D}} \cdot \mathrm{N}_{\mathrm{S}}
$$

The first level radio channel should be implemented on the transceivers that provide a range of communication within a radius of one domain up to 400-500 m (a typical linear configuration of 8 SAUs in increments of $50 \mathrm{~m}$ on two sides of the DCM for a domain with $\mathrm{N}_{M}=16$ ). The total transmission rate per domain (data stream per DCM) is determined by the digitization parameters of the seismic signal and the $\mathrm{N}_{M}$ number, which, in its turn, is determined by the radio conditions in the specific landscape conditions and the allowed frequency band in the selected range. Therefore, the minimum data transfer rate for a 16channel domain is 192000 bps.

The ISM 2.4 GHz band is targeted for a broad passband radio channel, which is inexpedient for RCh1. It is advantageous to apply the sub-gigahertz range, in which due to lower attenuation a lower output power of the radio signal is required. The choice of the LPD band allowed for telemetry (433-434 MHz) to be considered optimal if it is remote from residential areas (the range actively used by personal radios). In addition, it is applicable to work in an $868-869 \mathrm{MHz}$ range, which is narrowband but rarely used. When choosing the 
parameters of the RCh1 proceeding from the limited frequency resource (the width of the allowed bands is $700-1700 \mathrm{kHz}$ ), a search for a compromise solution between the two strategies is needed:

- To increase the number of DCMs, i.e., the number of adjacent RF channels, but to reduce the number of channels in the domain (the number of SAUs)

- To reduce the number of DCMs but increase the number of channels in the domain, i.e., increase the bandwidth of each RF channel.

The first option is relevant in the case of large attenuations (dense deciduous forest, difficult landscape, etc.). The second option is more economically advantageous and efficient in the case of minimal attenuation (for example, in an open field). It is also possible to configure the complex with domains with different numbers of SAUs.

It is reasonable to design the RCh1 network as a completely synchronous network within the domain, eliminating the need to apply any methods of competitive access to the channels such as CSMA) [5]. This will allow the most efficient use of the allocated frequency-time resource. In this case, synchronization of data transmission within the domain and the implementation of the TDMA method are assigned to the DCM.

The second level places higher demands on the radio network: with a cluster capacity of about 1000 measurement channels, the total data flow will be $12 \mathrm{Mbit} / \mathrm{s}$ at a sampling rate of $500 \mathrm{~Hz}$. The required transmission distance can be up to $50 \mathrm{~km}$ in the case of a linear configuration. The solution to this problem is proposed on the basis of the forwarding retransmission between the DCMs, while within a single cluster it can be deployed with multiple chains of transmission segments with a common center of data collection for the BS. The peak of information flow of the order of $10-20 \mathrm{Mbit} / \mathrm{s}$ will be in the DCM closest to the BS.

When implementing the RCh2, it is proposed to use IEEE $802.11 \mathrm{~b} / \mathrm{g}$ ISM $2.4 \mathrm{GHz}$ transceivers that fully comply with the requirements. To implement the forwarding retransmission method, it is necessary to develop an original network layer working over the standard IEEE $802.11 \mathrm{~b} / \mathrm{g}$ data link layer.

\section{Rch1 implementation}

Today, there are many economical single-chip transceivers for the sub-gigahertz range with programmable radio signal parameters. The majority of them support FSK, GFSK, GMSK frequency modulation types and their nominal data rates of up to $1 \mathrm{Mbps}$. Such transceivers fully comply with the specified requirements and can be the basis for the RCh1 implementation. Most commonly, chips implement a minimum of the data link layer functions, and the final developer has considerable freedom in the software implementation of MAC and LLC sub layers, network, and transport layers. One of such chips, Si446x, will be considered below [6].

\subsection{Single-chip sub-gigahertz Si446x transceiver specification}

Silicon Labs Si4468/7 chip is a sub-gigahertz FM transceiver with programmable parameters for organizing medium-range radio networks. Key features of the Si446x transceiver are:

- Frequency range of 142-1050 MHz;

- Modulation types: (G)MSK, OOK, (G)FSK, 4(G)FSK. 


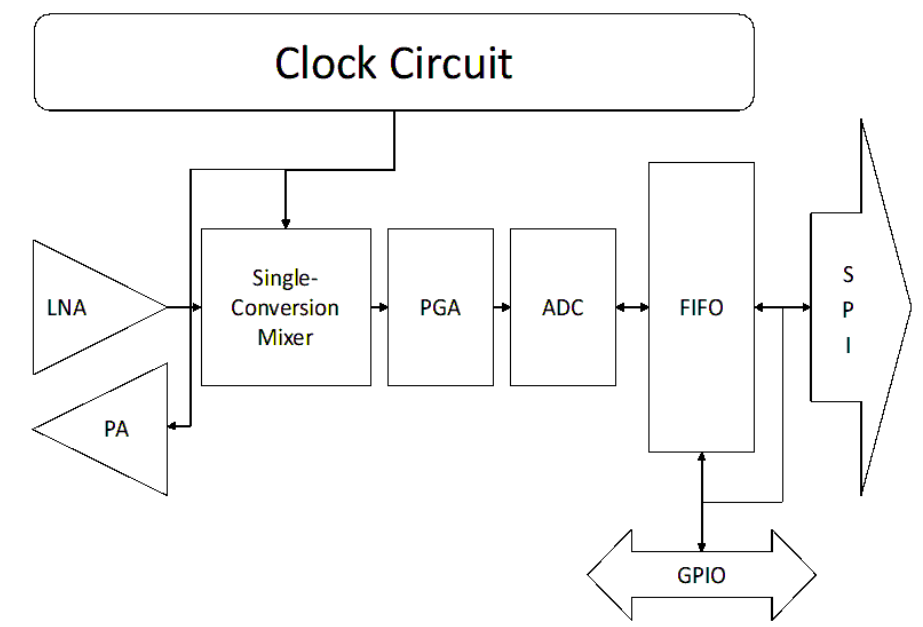

Fig. 2. Functional diagram of the transceiver Si4468/7.

The functional diagram of the transceiver is shown in Figure 2. The Si446x contains a low-noise differential amplifier (LNA), a single-conversion mixer to transform the OOK or 2/4-level FSK/GFSK modulated receive signal to a low IF frequency. The signal from the mixer is fed to a programmable gain amplifier (PGA). Also, the Si446x includes a highperformance $\mathrm{ADC}(\mathrm{ADC})$ and a signal processor, which are used to filter, demodulate, and process packets. A highly configurable packet handler allows to separate encoding/decoding of nearly any packet structure. Si4468 also contains a transmitter power amplifier (PA), a clock circuit, and a frequency synthesizer.

An SPI-interface controller is used to connect to an external microcontroller. The chip also contains a built-in temperature sensor, a power tracking circuit (POR), and four input/output ports (GPIO1-4) with various functions assigned to them: control of an external antenna switch, output of various states transceivers, etc. The transceiver includes the transmitting and receiving FIFO-buffers. The microcircuit allows creating FDMA and TDMA radio networks at the program layer.

\subsection{The physical layer of the RCh1}

To study the possibility of using a transceiver to build RCh1, a radio module based on the Si4468 transceiver was developed. Features of the developed radio module are:

- Frequency band of $433.075-434.775 \mathrm{MHz}$

- Output power programmable from 1 to $50 \mathrm{~mW}$

- Frequency modulation

- Maximum transfer rate of $1 \mathrm{Mbps}$

- Power supply of $3.3 \mathrm{~V}$

- SPI interface

- Operating temperature range -40 to $+40^{\circ} \mathrm{C}$.

It is possible to select radio channel parameters such as modulation type (GFSK, 4GFSK), preamble length, transmission rate $(32-512 \mathrm{kbit} / \mathrm{s})$, frequency modulation index $(\mathrm{m}=0.5, \mathrm{~m}=0.75)$ radio channel numbers.

In this work, we consider the example of radio channel implementation with a nominal transmission rate of $256 \mathrm{kbit} / \mathrm{s}$ using a transceiver. To use the frequency resource more effectively, it is proposed to use 4GFSK modulation. The use of 4GFSK modulation using six 
radio channels in the selected frequency band for the transmission rate, since the use of a Gaussian filter reduces the side lobes of the spectrum and also significantly increases the rate of reduction of the spectrum of the GFSK signal compared to FSK signals.

However, with GFSK modulation, symbol-to-symbol interference increases. Experiments showed that the most optimal value of the BT parameter of the Gauss filter is 0.5. A further decrease of BT is impractical because it increases the symbol-error probability.

Figure 3 shows a 4GFSK signal spectrum with frequency modulation index $\mathrm{m}=0.5 \mathrm{ob}-$ served in practice at the output of the radio module based on the Si4468 transceiver. The width of the signal's spectrum by level $-25 \mathrm{~dB}$ with a modulation index of 0.5 was $357 \mathrm{kHz}$.

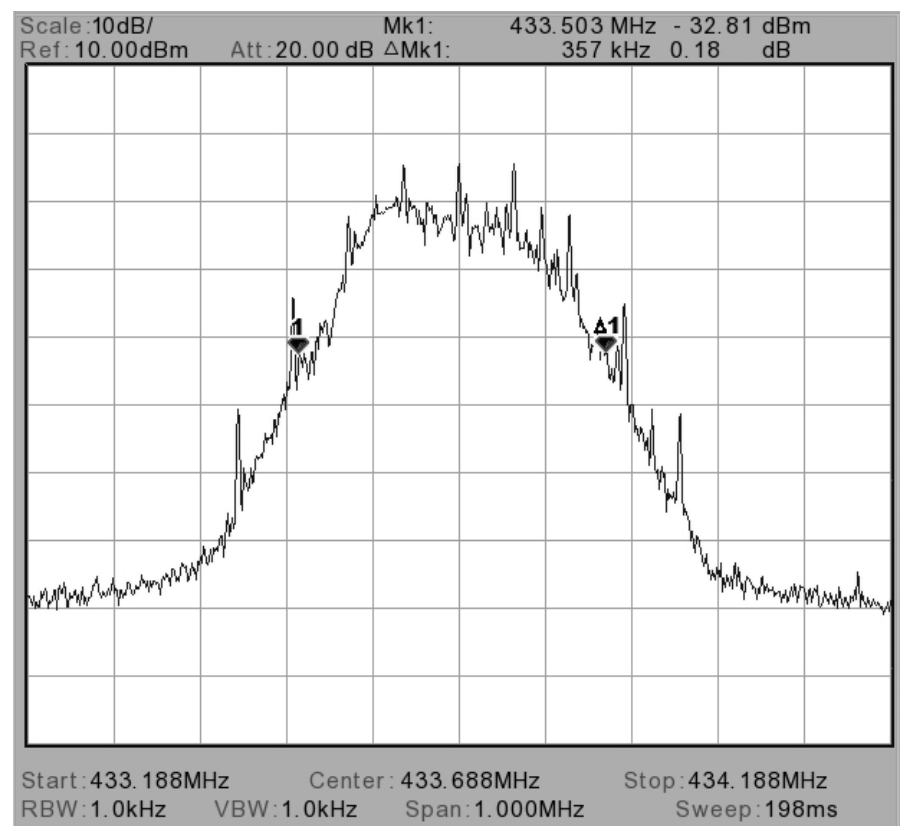

Fig. 3. A practical spectrum of 4GFSK signal with a modulation index of 0.5 .

\subsection{Data link layer of the RCh1}

As stated above, it is reasonable to design the RCh1 network as a completely synchronous network within the domain.

For the practical implementation of a synchronous system, it is necessary to determine the dimension of the node address, the most advantageous length, and the format of the frame. It should be noted that the implementation of any method of competitive access to the channel is not obligatory.

The proposed frame structure is shown in Figure 4. The preamble is a $1010 \ldots$ bit sequence, the length of which is selected on the basis of the modulation type. For a 4GFSK signal, the recommended preamble length is 10 bytes [7].

The length of the sync word is two bytes. The transceiver has an ability of computing CRC of each field independently, or the CRC of the entire frame. In our case, two fields are used to check the integrity of the full frame. The maximum length of all fields is determined by the size of 64-byte transmit and receive FIFO buffers, into which the transmitted and received data are loaded. It is possible to combine both buffers into one buffer with a length of 129 bytes, which allows to increase the frame length. 


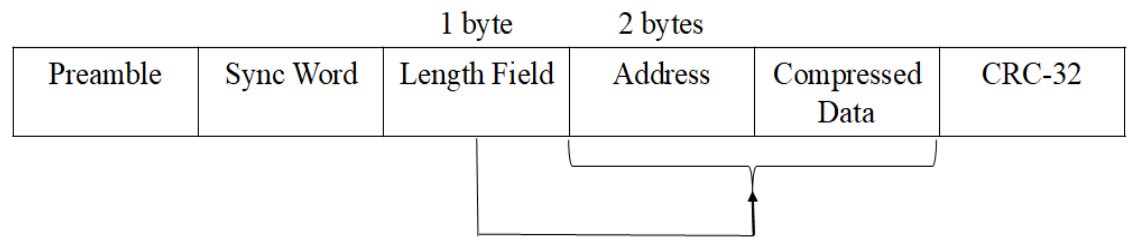

Fig. 4. The common frame format of the radio network.

One of the benefits of using the Si446x transceiver is the feasibility of receiving a frame without knowing the data field length, which is optimal since there is no need to transmit packets of equally large length, even if the size of the data itself is small. In such a scenario, the transmitted packet should contain up to two bytes, defining the length of the variable field. In this case, it is necessary to include the length of the remaining part of the second field (payload) into the first field of the frame. These length bytes should be located in the packet before the variable-length field.

Otherwise, the recipient receiver will not have the information to allocate the received data bytes. In our case, the first field with a length of one byte contains the total length of the second field. The second field contains the payload, the length of which is not known in advance.

Registers PKT_LEN, PKT_LEN_FIELD_SOURCE, PKT_FIELD_1_LENGTH, PKT_FIELD_2_LENGTH of Si446 $\bar{x}$ microcircuit is used to set the number and size of fields and to receive a frame, whose length is stated in the first field. The position of the length field and the number of fields in the package is set in the PKT_LEN, PKT_LEN_FIELD_SOURCE registers. The maximum length of fields 1 and 2 is set in the PKT_FIELD_1_LENGTH and PKT_FIELD_2_LENGTH registers [6]. At the end of the frame, the CRC-32 checksum is calculated.

\section{Seismic data compression}

As shown above, the minimum transfer rate for a domain on 16 channels is $192000 \mathrm{bps}$. So, it is an urgent task of reducing the amount of data transmitted via the radio channel to optimize the load on the radio network and reduce power consumption. The ARM Cortex-M4 microcontroller solves this problem, receiving seismic data and compressing them. To implement compression, we used the Miniz library [8] that was modified for use with a microcontroller. This library contains an implementation of the LZ77 compression algorithm and Huffman entropy coding. It was possible to achieve the values of the compression ratio of 1.5-1.8 for the seismic signal.

The flowchart of the algorithm for accumulating and compressing data is shown in Figure 5. Data is accumulated in the ring buffer of the microcontroller as soon as the first fraction of the buffer is filled, the compression subroutine started and accumulation continue; the same is accomplished with the second part of the buffer. 


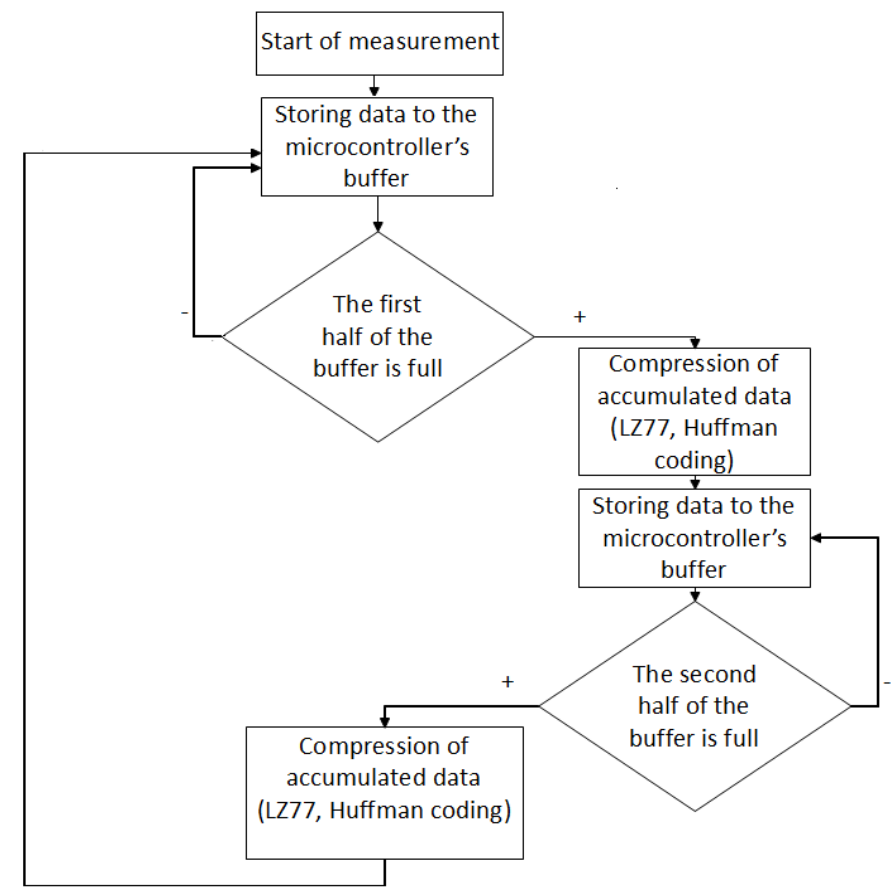

Fig. 5. The algorithm for accumulating and compressing data.

The compressed seismic data is transmitted as a radio packet as shown in Figure 4.

\section{Test results}

Field tests of the complex were conducted in the open area and in the forest, the configuration of the arrangement of the 16 SAUs and the DCM is shown in Figure 6.

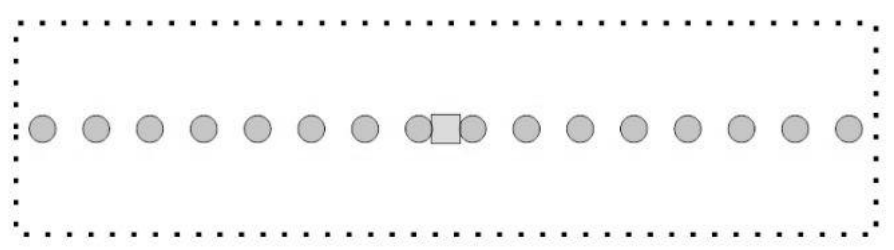

Fig. 6. The arrangement of the modules in the field tests.

In field tests, the range of confident radio communication of the presented radio modules was determined with the following settings:

- frequency channels,

- polling period; $200 \mathrm{~ms}$,

- frequency range $433 \mathrm{MHz}$,

- modulation type 4GFSK,

- maximum permissible transmitter power,

- transmission speed $350 \mathrm{kbit} / \mathrm{s}$. 
The range of reliable radio communication of the radio modules $(90-100 \%$ received packets) under the given conditions is presented in Table 1.

Table 1. Field test results

\begin{tabular}{ccc}
\hline Test conditions & Range, $\mathrm{m}$ \\
$\begin{array}{c}\text { in condition small elevations } \\
\text { in line of sight }\end{array}$ & In the field & 400 \\
$\begin{array}{c}\text { dense forest or tall grass } \\
\text { forest aisle }\end{array}$ & in the woods & 200 \\
\hline
\end{tabular}

\section{Conclusion}

The architecture of the complex considered in this work was implemented on the basis of the Si446x programmable transceiver. Radio channel parameters such as bit rate, modulation index, and type and level of radiated power are configured based on the tasks to be solved in the selected frequency range. The applied algorithm of the seismic data compressing allows to optimize the load on the radio network.

The comparison of existing and proposed solutions is given in Table 2.

Table 2. Comparative analysis of existing and proposed solutions.

\begin{tabular}{lccc}
\hline \multicolumn{1}{c}{ Characteristic } & \multicolumn{1}{c}{ RT2 } & SCOUT & $\begin{array}{c}\text { Proposed } \\
\text { architecture }\end{array}$ \\
\hline Frequency range & $2,4 \mathrm{GHz}$ & Wi-Fi, 2,4 GHz & $433 \mathrm{MHz}$ \\
Data transmission method & Retransmitting & Retransmitting & $\begin{array}{c}\text { Synchrnous } \\
\text { Available }\end{array}$ \\
Real-time mode & Available & Not available & 6 \\
Number of channels & 13 & 13 & Up to 30 days \\
Run time of the module & Up to 25 days & Up to 25 days & $350 \mathrm{kbit} / \mathrm{s}$ \\
Transmission speed & $2 \mathrm{Mbit} / \mathrm{s}$ & $2 \mathrm{Mbit} / \mathrm{s}$ & $500 \mathrm{~m}$ \\
Transmission range & $200 \mathrm{~m}$ & $200 \mathrm{~m}$ & \\
\hline
\end{tabular}

Using the sub-gigahertz range allows to achieve a longer communication range than in existing complexes and reduces the level of power consumption, which in its turn allows to increase the operating time. On the other hand, this causes a decrease in the number of channels and in the transmission speed, but these values are sufficient to implement a realtime system within this architecture.

\section{References}

1. V.I. Popov, Basics of cellular communication of gsm standard. (Moscow: eco-trends).

2. A.V. Cherepovskiy, Land seismic of new technological level. (Moscow: EAGE Geomodel 2016).

3. Wireless Seismic, Recording system with real-time data transmission RT2. Datasheet. (Sugar Land: Wireless Seismic, 2017). 
4. D. K. Nurgaliev, O. N. Sherstyukov, E. Y. Ryabchenko, E. V. Danilov, A. D. Smolyakov, L. S. Murtazina Multi-Level Radio Network Architecture For Seismic Data Acquisition System, Russian Open Conference on Radio Wave Propagation, 403-405 (2019).

5. O. N. Sherstyukov, E. Y. Ryabchenko, E. V. Danilov, A. D. Smolyakov L. S. Murtazina Sub-Gigahertz Band Sensor Radio Network, Russian Open Conference on Radio Wave Propagation, 406-409 2019.

6. Silicon Laboratories Inc. Packet handler operation for Si446x RFICs. Application Note AN626. (Austin: Silicon Laboratories Inc. 2013).

7. Silicon Laboratories Inc. Si4468/7 - High-performance, low-current transceiver. Datasheet. (Austin: Silicon Laboratories Inc 2014).

8. R. Geldreich Miniz library (2011). 